\title{
Damit Ihre Kinder nicht unsere Patientinnen und Patienten werden!
}

Wir haben als Ärztinnen und Ärzte alle täglich mit Patienten zu tun, welche an Lungen-, Herz-, Gefäss-, Tumorsowie anderen tabakbedingten Krankheiten leiden. Und wir versuchen, ihnen so gut wie möglich zu helfen. Hinter diesen Krankheiten stecken Leid und Einschränkungen für die Betroffenen, aber auch für ihre Angehörigen. Ein überwiegender Anteil dieser Krankheiten steht in direktem Zusammenhang mit dem Tabakkonsum und könnte daher vermieden werden. Die Zahl an chronisch obstruktiven Lungenerkrankungen, Herzinfarkten und Lungentumoren könnte künftig massiv reduziert werden.

Vor diesem Hintergrund ist es unsere Aufgabe, uns für gesetzliche Regelungen betreffend Tabakprodukte einzusetzen, die verhindern, dass Menschen dazu verführt werden, mit dem Rauchen anzufangen. Es geht nicht darum, den Tabakkonsum generell zu verbieten. Das Ziel sollte sein, die Vermarktung dieser Produkte einzuschränken, damit insbesondere junge Menschen den Tabakkonsum nicht als banal erleben, wie dies heute der Fall ist: Online-Wettbewerbe, Sponsoring von Veranstaltungen, direkte und personalisierte Promotion, an festlichen Anlässen geschickt aufgestellte Zigarettenautomaten - jedes Mittel ist recht, um Tabak bei Jugendlichen bekannt zu machen, welche morgen unsere Patientinnen und Patienten sein werden. Mar-

Prof. Dr. med. Paola Gasche-Soccal

Schweizerische Gesellschaft für Pneumologie (SGP) Präsidentin

Korrespondenz:

PD Dr. med.

Macé M. Schuurmans Ko-Präsident Arbeitsgruppe Tabakprävention/Rauch-

stopp

Schweizerische Gesellschaft

für Pneumologie (SGP)

Chutzenstrasse 10

CH-3007 Bern

Tel. 0313782030

mace.schuurmans[at]usz.ch

Elena Strozzi Bereichsleiterin Politik und Prävention - Mitglied der

Geschäftsleitung

Lungenliga Schweiz

Chutzenstrasse 10

CH-3007 Bern

Tel. 0313782038

e.strozzi[at]lung.ch
Prof. Dr. med. Béatrice Amann-Vesti

Schweizerische Gesellschaft für Angiologie (SGA)

Präsidentin

Dr. med. Urs Kaufmann

Schweizerische Gesellschaft für Kardiologie (SGK)

Präsident

Dr. med. Jürg Schlup

Verbindung der Schweizer Ärztinnen und Ärzte FMH

Präsident

Prof. Dr. med. Raffaele Rosso FACS FRCS

Schweizerische Gesellschaft für Chirurgie (SGC)

Präsident ketingstrategien werden immer personalisierter und gezielter und erreichen Jugendliche direkt und überall Oder wie ist es sonst zu erklären, dass die Anzahl Rauchender in der Schweiz nicht mehr zurückgeht, obwohl unsere Patientinnen und Patienten täglich wegen des Tabakkonsums sterben? Beginnen Jugendliche vor dem 20. Altersjahr zu rauchen, steigt ihr Risiko massiv, später tabakabhängig zu werden.

Wir möchten Sie bitten, die Gelegenheit wahrzunehmen, mit der Erarbeitung des Tabakproduktegesetzes den Tabak auf das zu beschränken, was er sein sollte: ein stark gesundheitsgefährdendes Produkt, das nicht beworben werden darf und dessen Handel internationalen Regulierungen unterstellt sein muss. Deshalb verlangen wir ein umfassendes Verbot von Marketing, Promotion und Sponsoring für Tabakprodukte, sowohl in traditionellen Medien als auch im Internet, an Veranstaltungen, Verkaufsorten, und ein Verbot von Zigarettenautomaten. Zahlreiche andere Länder haben solche Regelungen bereits eingeführt - die Schweiz soll sich diesen anschliessen.

Wir bitten Sie, durch Ihre Unterstützung Ihre Verantwortung wahrzunehmen. Damit die Kinder von heute nicht unsere Patientinnen und Patienten von morgen werden.

Dr. med. Jürg Nadig

Schweizerische Gesellschaft für medizinische Onkologie (SSMO) Präsident

\section{Prof. Dr. med. Jürg Hammer}

Schweizerische Gesellschaft für pädiatrische Pneumologie (SGPP) Präsident

Dr. med. Nicole Pellaud

Schweizerische Gesellschaft für Pädiatrie (SGP)

Präsidentin

Prof. Dr. med. Jean-Michel Gaspoz

Schweizerische Gesellschaft für Allgemeine Innere Medizin (SGAIM) Ko-Präsident

Dr. med. François-Gérard Héritier

Schweizerische Gesellschaft für Allgemeine Innere Medizin (SGAIM) Ko-Präsident 